\title{
Observação de uma tempestade geomagnética sobre a cidade de Manaus - Brasil (25 Outubro 2011) com dados de GPS (NovAtel).
}

Leonaldo de Menezes Moraes; Newton Silva de Lima ; Alan dos Santos Ferreira; Naiana Lopes Ramos ; Matheus Elia Hage Rodrigues; Lenidia Renz; Rodrigo de Souza Machado; Elaine Chrystine da Silva Albuquerque.

Centro Universitário Luterano de Manaus - ULBRA

Av. Carlos Drummond de Andrade, 1460 (Japiim II - Conj. Atílio Andreazza)

CEP 69077-730 Tel. (92) 3616-9800 Manaus Amazonas - Brasil

e-mail: naiana.lopes.r@gmail.com

Copyright 2014, SBGf - Sociedade Brasileira de Geofísica

Este texto foi preparado para a apresentação no VI Simpósio Brasileiro de Geofísica, Porto Alegre, 14 a 16 de outubro de 2014. Seu conteúdo foi revisado pelo Comitê Técnico do VI SimBGf, mas não necessariamente representa a opinião da SBGf ou de seus associados. É proibida a reprodução total ou parcial deste material para propósitos comerciais sem prévia autorização da SBGf.

\section{Resumo}

Estudo realizado sobre uma tempestade geomagnética, com a utilização do GPS - NovAtel, na cidade de Manaus-AM(2,9 $\mathrm{S}, 60 \mathrm{~W})$, realizado a partir da aquisição de dados referentes ao conteúdo eletrônico total ("TEC"em inglês) da intensidade da Dst (Disturbance Storm Time), ocorrida após um "solar flare" no dia 23 Outubro de 2011 com ejeção de massa coronal no espaço interplanetário ("CME" em inglês) desenvolvendo uma tempestade magnética que acoplou-se na magnetosfera terrestre, consequentemente produzindo auroras e distúrbios ionosféricos viajantes na direção equatorial terrestre, sendo visualizado na ionosfera tropical sobre a cidade de Manaus (Brasil) através da degradação do sinal GPS referente ao mês de Outubro do ano de 2011, no ciclo solar \#24, sua real intensidade, período de ocorrência e dias de excitação na ionosfera sobre o alvo foram representados pela série temporal e em Transformada em Ondeleta de "Morlet".

\section{Introdução}

O trabalho realizado na aquisição de dados, relativos à cidade de Manaus (Latitude 2,9 $9^{\circ} \mathrm{S}$ e Longitude $60,0^{\circ}$ W), que devido a sua localidade, próximo aos equadores magnético e geográfico foi feito com base nos estudos realizados na análise do banco de dados coletados pelo GPS - NovAtel e na série temporal de Dst (WDC/DstKyoto, 2011) de monitoramento do Ciclo Solar com o objetivo de compreender a influência da ejeção de massa coronal sobre a magnetosfera terrestre. $\mathrm{E}$ em relação às alterações das propriedades físicas da ionosfera, através do conteúdo eletrônico total (TEC), ou seja, a quantidade de cargas eletrônicas livres por unidade de área $\left(10^{16}\right.$ $\mathrm{e} / \mathrm{m}^{2}$ ), uma característica da ionosfera proveniente do processo pelo qual a radiação ultravioleta (raios UV) energiza os elétrons de valência de tal modo que os átomos dos gases atmosféricos ionizam-se. (Silva, 2006).

O fenômeno em alta atmosfera chamado de bolhas de plasma ocorre durante a transição dia-noite devido ao pico pré-reverso, como consequência, o campo elétrico local é invertido e ocasiona o cisalhamento da região ionosférica resultando em duas partes (mais densa sobre menos densa). A partir daí, qualquer instabilidade na região atmosférica propicia o fenômeno de bolhas de plasma que ocorre somente no período noturno, comum na Amazônia.

O índice Dst indica a ocorrência de tempestades geomagnéticas no mês trabalhado (Fig. 1).

A análise do conteúdo eletrônico total ("TEC" em inglês) permite a visualização da degradação do sinal GPS, consequentemente a formação de bolhas de plasma. (Fig. 3).

\section{Resultados}

O índice Dst mostra que no mês de Outubro mostra algumas tempestades geomagnéticas de baixa

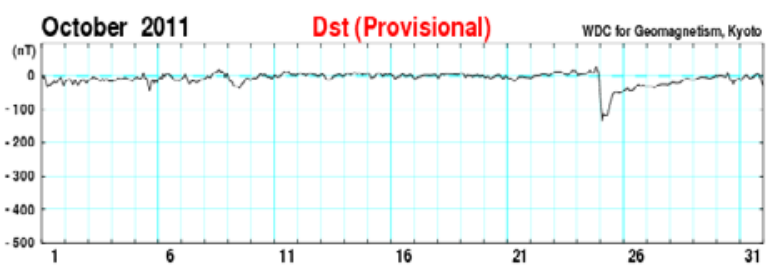

intensidade no período dos dias 4 e 8. A tempestade geomagnética de maior intensidade ocorreu no dia 25 , como mostra a Fig 1.

Fig 1 - Índice Dst do mês de Outubro de 2011.

(Fonte:http://wdc.kugi.kyoto-u.ac.jp/dstdir/)

A Fig. 2 representa a série temporal para o índice de Dst e Transformada em Ondeleta de Morlet. Sendo possível visualizar uma tempestade geomagnética de baixa intensidade (dias 4 e 8) e a de alta intensidade ocorrida no dia 25 , todas estão dentro do cone de confiabilidade.

Nota-se que no dia 25 de Outubro houve bastante bolhas de plasma no período de $20 \mathrm{~h}$ (dia 24) até $6 \mathrm{~h}$ (dia 25), degradando o sinal de GPS. (Fig. 3). 


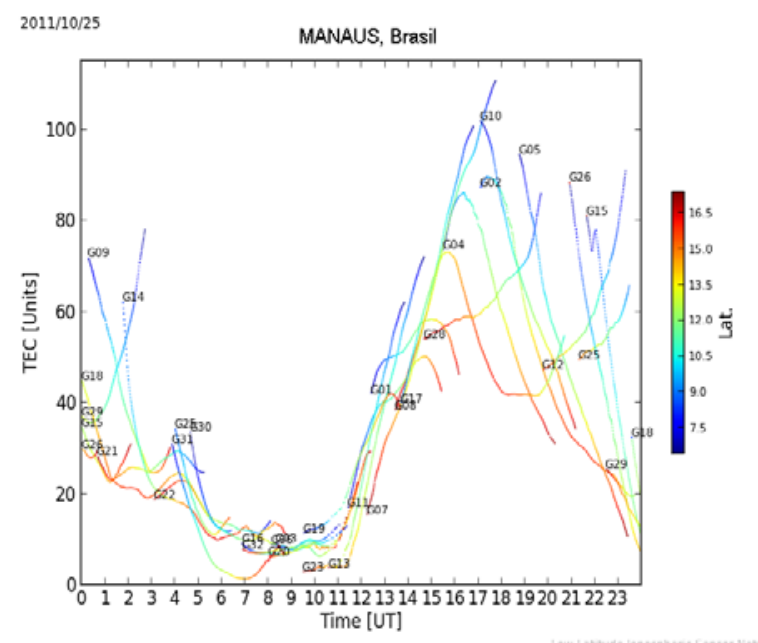

Fig 3 - Representa o conteúdo eletrônico total (TEC), percebe-se a formação de bolhas de plasma durante 0 período noturno (até às 6h). (Fonte: http://lisn.igp.gob.pe/data/).

\section{Discussão e Conclusões}

A tempestade geomagnética ocorrida no dia 25 de Outubro de 2011 foi de alta intensidade, podendo ser observado pela série temporal de Dst relacionada com o gráfico de espectro de potência em Ondeleta de Morlet, que seu período durou por volta de 4 à 6 dias dentro do cone de confiabilidade, como mostra a Fig. 2. As bolhas de plasma são observadas junto com a degradação do sinal GPS no gráfico representado pela Fig. 3, durante o período noturno.

\section{Agradecimentos}

Agradecemos nesse trabalho ao apoio dos professores orientadores: Newton Silva de Lima e Alan dos Santos Ferreira; a ULBRA Manaus pela estrutura, pelos recursos. a Fundação de Amparo à Pesquisa do Estado do Amazonas; a UNIVAP; a LISN e Estação de Kyoto no Japão pelos dados TEC e Dst, respectivamente.

\section{Referências}

\section{http://lisn.igp.gob.pe}

http://wdc.kugi.kyoto-u.ac.jp/dst provisional

Silva, A, V. R., 2006. Nossa Estrela: O Sol. $1^{\mathrm{a}}$ ed., Livraria da Física, São Paulo, Brasil.

Turner, F. J. \& Werhoogen, J., 1960. Igneous and metamorphic petrology. 2nd ed., McGraw Hill, New York, USA. 


\section{ANEXOS}

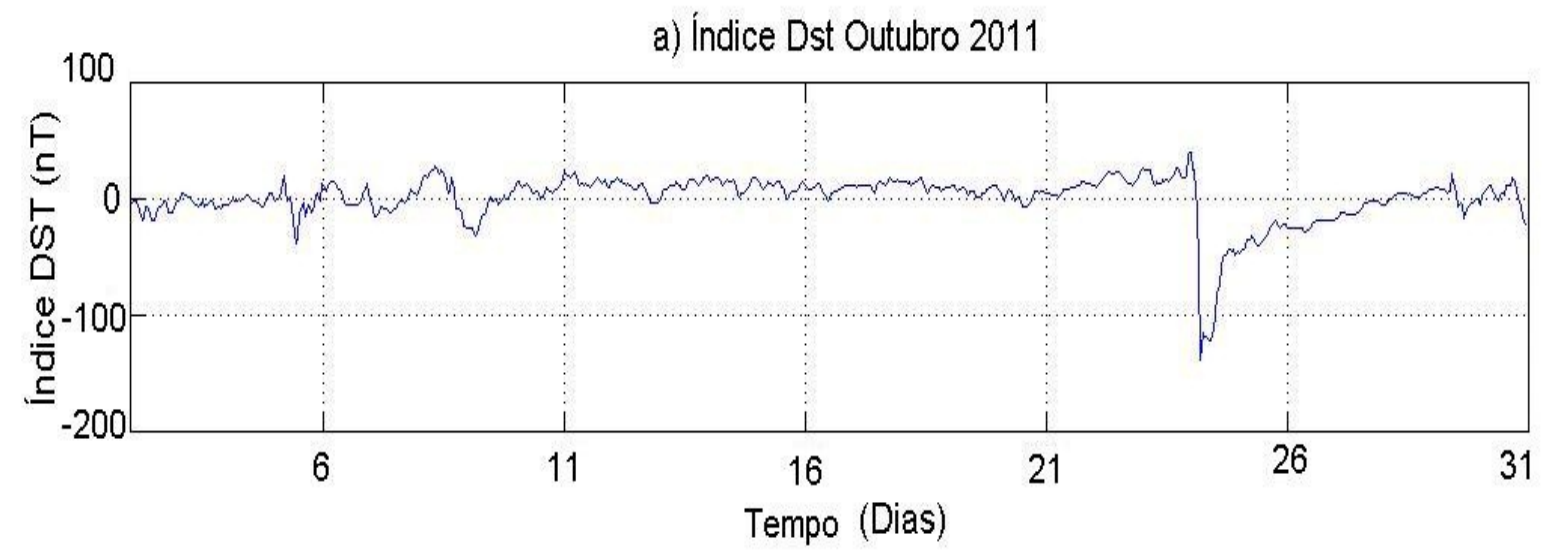

b) Índice DST em nT do Espectro de Potência em Ondeleta
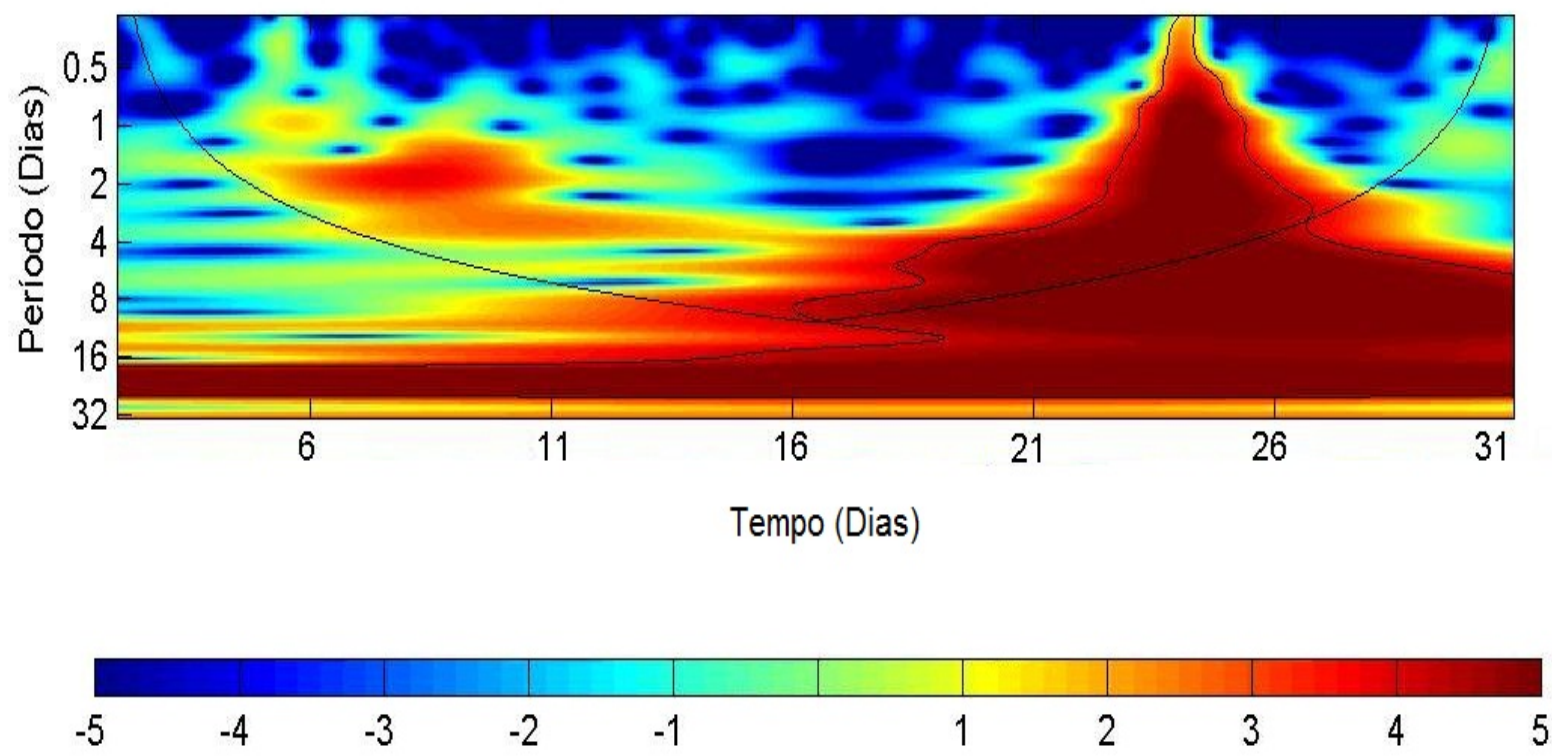

Figura 2 - Série temporal de Dst do mês de Outubro relacionada com o gráfico do espectro de potência em Ondeleta de Morlet. 
Observação de uma tempestade geomagnética sobre a cidade de Manaus

\begin{abstract}
-
\end{abstract}

